\title{
The contribution of clinical assessments to the diagnostic algorithm of pulmonary embolism
}

\author{
Onur Turan ${ }^{1, A-F}$, Deniz Turgut ${ }^{2, A-C}$, Turkan Gunay ${ }^{3, B-D}$, Erkan Yilmaz ${ }^{2}, A-C$, Ayse Turan ${ }^{4, D-F}$, Atila Akkoclu ${ }^{5, A-C}$ \\ ${ }^{1}$ Chest Disease Department, Faculty of Medicine, Izmir Katip Celebi University, Ataturk Training and Research Hospital, Karabaglar, İzmir, Turkey \\ ${ }^{2}$ Radiodiagnostics Department, Faculty of Medicine, Dokuz Eylul University, Inciraltı, İzmir, Turkey \\ ${ }^{3}$ Public Health Department, Faculty of Medicine, Dokuz Eylul University, Inciraltt, İzmir, Turkey \\ ${ }^{4}$ Menemen State Hospital, Menemen, İzmir, Turkey \\ ${ }^{5}$ Chest Disease Department, Faculty of Medicine, Dokuz Eylul University, Inciralt, İzmir, Turkey \\ A - research concept and design; $\mathrm{B}$ - collection and/or assembly of data; $\mathrm{C}$ - data analysis and interpretation; \\ $D$ - writing the article; $E$ - critical revision of the article; $F$ - final approval of article
}

\section{Address for correspondence}

Onur Turan

E-mail: onurtura@yahoo.com

\section{Funding sources}

none declared

\section{Conflict of interest}

none declared

Received on 0ctober 26, 2014

Revised on November 19, 2014

Accepted on December 15, 2014
DOI

10.17219/acem/35106

\section{Copyright}

Copyright by Author(s)

This is an article distributed under the terms of the

Creative Commons Attribution Non-Commercial License

(http://creativecommons.org/licenses/by-nc-nd/4.0/)

\begin{abstract}
Background. Pulmonary thromboembolism (PE) is a major disease in respiratory emergencies. Thoracic CT angiography (CTA) is an important method of visualizing PE. Because of the high radiation and contrast exposure, the method should be performed selectively in patients in whom PE is suspected.

Objectives. The aim of the study was to identify the role of clinical scoring systems utilizing CTA results to diagnose PE.

Material and methods. The study investigated 196 patients referred to the hospital emergency service in whom PE was suspected and CTA performed. They were evaluated by empirical, Wells, Geneva and Miniati assessments and classified as low, intermediate and high clinical probability. They were also classified according to serum D-dimer levels. The sensitivity, specificity, positive predictive value (PPV) and negative predictive value (NPV) were calculated and evaluated according to CTA findings.

Results. Empirical scoring was found to have the highest sensitivity, while the Wells system had the highest specificity. When low D-dimer levels and "low probabilty" were evaluated together for each scoring system, the sensitivity was found to be 100\% for all methods. Wells scoring with a cut-off score of 4 had the highest specificity (56.1\%).

Conclusions. Clinical scoring systems may be guides for patients in whom PE is suspected in the emergency department. The empirical and Wells scoring systems are effective methods for patient selection. Adding evaluation of D-dimer serum levels to the clinical scores could identify patients in whom CTA should be performed. Since CTA can only be used conservatively, the use of clinical scoring systems in conjunction with D-dimer levels can be a useful guide for patient selection.
\end{abstract}

Key words: pulmonary embolism, clinical scoring systems, Wells score 
Pulmonary embolism (PE) is a disease with high morbidity and mortality if not treated, and early diagnosis is of vital importance. ${ }^{1}$ However, the symptoms, clinical and laboratory findings have low sensitivity and specificity in confirming a diagnosis of $\mathrm{PE}^{2}$ Diagnostic tests to confirm or rule out PE may be expensive and are not always available. Thoracic CT angiography (CTA) is an important method for direct visualization of the embolism, but should be performed selectively in patients in whom PE suspected, because of high radiation and contrast exposure. ${ }^{3}$ Other invasive methods such as pulmonary angiography involve the risk of some complications, limiting their routine usage in these patients. ${ }^{4}$

The combination of medical history, physical examination and some simple laboratory parameters have been used to predict the probability of PE. ${ }^{5,6}$ Empirical scoring is one of these systems, and it mostly shows the clinical decision of the clinician. ${ }^{7}$ The Wells criteria are one of the most objective predictive systems, categorizing patients into low, intermediate and high risk for PE. ${ }^{8}$ Geneva scoring is also based on objective variables including arterial blood gas parameters. ${ }^{9}$ The Miniati scoring system, another pre-test, has similar results as ventilation-perfusion scintigraphy (V/Q scan) in predicting the presence of PE. ${ }^{10}$ After appropriate selection of patients that meet the criteria for further evaluation for PE, these pre-tests can reduce unnecessary imaging and radiation exposure while optimizing PE treatment. ${ }^{6}$

In addition to clinical scoring systems, D-dimer levels are used where deep venous thrombosis (DVT) and PE are suspected. A normal serum D-dimer level may exclude a diagnosis of PE. ${ }^{11}$ Clinical scores combined with D-dimer levels have been shown to be useful for excluding PE. ${ }^{6}$

Since clinical scoring systems can serve as a guide for the prediction of PE, the aim of this study was to identify their role in correlation with CTA findings for diagnosing PE.

\section{Material and methods}

The study involved 196 patients admitted to the Dokuz Eylul University emergency department in 2009 in whom CTA was performed due to suspected PE. The study was approved by the university ethics committee.

The hospital files of the 196 patients were investigated retrospectively. All the data, including demographics and medical histories, clinical signs and symptoms, laboratory and radiographic test results (arterial blood gas [ABG] analysis, enzyme-linked immunosorbent assay [ELISA], serum D-dimer measurement by the turbidimetric method, electrocardiography [ECG], chest X-ray, lower extremity venous Doppler ultrasound) were recorded. The clinical scores were evaluated according to empirical scoring (Table 1a), the Wells criteria (Table 1b), Geneva scoring (Table 1c) and the Miniati system (Table 1d). ${ }^{7-10}$ The patients were classified as low, intermediate or high clinical probability of $\mathrm{PE}$ according to their clinical scores, and were also categorized as "PE likely" or "PE unlikely" by a modified Wells score with a cut-off score of 4 points.

Table 1a. Empirical scoring system ${ }^{7}$

\begin{tabular}{|ll|}
\hline 1 & Righ probability \\
\hline 2 & Unexplained dyspnea, tachypne, or pleuritic pain present \\
3 & Unexplained radiographic or gas exchange abnormality present \\
\hline 4 & Neither high nor low clinical probability Intermediate probability \\
\hline 5 & Risk factor not present \\
\hline 7 & Radiographic or gas exchange abnormality possibly present but explainable by another condition \\
\hline
\end{tabular}

Table 1b. Wells scoring system ${ }^{8}$

\begin{tabular}{|lc|}
\hline Clinical signs and symptoms of DVT (min of leg swelling and pain with palpation of the deep veins) & +3.0 \\
PE as or more likely than an alternative diagnosis & +3.0 \\
Heart rate greater than 100 & +1.5 \\
Immobilization or major surgery in the previous 4 weeks & +1.5 \\
Previous DVT/PE & +1.5 \\
Hemoptysis & $+1.0+1.0+$ \\
Active cancer (treatment ongoing or within the last 6 months or palliative) & +1.0 \\
\hline
\end{tabular}

A score of less than 2.0 indicates low pre-test probability, a score between 2.0 and 6.0 indicates intermediate pre-test probability and a score of more than 6.0 indicates high pre-test probability. 
Table 1c. Geneva scoring system ${ }^{9}$

\begin{tabular}{|lcc|}
\hline Age in years & $60-79$ & +1 \\
& $\geq 80$ & +2 \\
Previous DVT or PE & & +2 \\
Heart rate greater than 100/min & +1 \\
Recent surgery & +3 \\
$\mathrm{~Pa} \mathrm{CO}, \mathrm{kPa}$ & $<4.8$ & +2 \\
& $4.8-5.19$ & +1 \\
$\mathrm{~Pa} \mathrm{O}_{2}, \mathrm{kPa}$ & $<6.5$ & +4 \\
& $6.5-7.99$ & +3 \\
& $8-9.49$ & +2 \\
& $9.5-10.99$ & +1 \\
Chest X-ray & plate-like atelectasis & +1 \\
& elevation of hemidiaphragm & +1 \\
\hline
\end{tabular}

Geneva Score: 0-4: Iow probability, 5-9: intermediate probability, > 9: high probability. were the most frequent symptoms. Tachypnea (42.3\%) and tachycardia (14.8\%) were the most frequent physical examination findings. Immobilization (41\%), the presence of malignancy (18\%) and surgical history (16\%) were the most frequent risk factors for PE in the study group.

Doppler ultrasound was performed in 51 of 106 patients with suspected deep vein thrombosis (DVT); DVT was confirmed in 21 of the 51 patients $(41.2 \%$ of those in whom Doppler ultrasound was performed). Transthoracic echocardiography (TTE) revealed abnormal echocardiographic features (seen in PTE) in 9 out of 16 patients in whom TTE was performed.

Arterial blood gas analysis was carried out $88.8 \%$ of the patients in the study group; it demonstrated hypoxia and hypocapnia in 55 patients (31.6\%), only hypoxia in 48 (27.6\%), and only hypocapnia in 43 (24.7\%).

Table 1d. Miniati clinical scoring system ${ }^{10}$

High probability

Acute dyspnea, chest pain or syncope (min. one symptom present) not explainable by another condition, with:

Electrocardiographic signs of right heart failure, pulmonary oligemia, amputation of hilar artery or pulmonary infarction (min. 2 findings present)

Intermediate probability

One symptom present without electrocardiographic signs and radiological findings

Low probability

No symptom present or any possible diagnosis (such as COPD or pneumonia) that can be an explanation for these symptoms

The patients' CTA images and the reports of the radiodiagnostics department were reviewed. They were divided into 2 groups according to whether PE was present or not. The patients with positive radiological findings for PE were categorized as the PE group. The parameters of each scoring system were categorized into 2 groups depending on the presence of PE.

The clinical scoring of the patients was analyzed using SPSS 11.0 software (IBM Corp., Armonk, USA). Sensitivity, specificity, positive and negative predictive values were calculated and compared. The combination of a negative D-dimer level and a clinical score of low probability was treated as another group and compared with the rest of the patients regarding the presence or absence of PE. $X^{2}$ statistics were used to compare categorical variables. The statistical significance of any differences was evaluated by the non-parametric Mann-Whitney U-test when the data were from a highly non-normal distribution, and by the $\mathrm{T}$ test when the data were from a normal distribution. The area under the reciever operating characteristic (ROC) curve was calculated for each scoring system.

\section{Results}

The study group included 107 males (54.6\%) and 89 females (45.4\%) suspected of PE; their mean age was $64.18 \pm 17.44$. Dyspnea (73\%) and chest pain (45.9\%)
D-dimer levels were measured in 141 patients and found to be elevated in 117 patients (83\%), with a cut-off level of $500 \mathrm{ng} / \mathrm{mL}$.

PE was diagnosed by CTA in 39 patients (19.9\%). The mean age of these patients was $68.85 \pm 15.56$, and there was no statistically significant difference between ages of the patients in groups with or without PE $(\mathrm{p}=0.062)$. The patients with PE included 13 men $(33.3 \%)$ and 26 women $(66.7 \%)$. Out of the total 196 patients in whom PE was suspected, $12.1 \%$ of the men (13/107) and $29 \%$ of the women $(26 / 89)$ had PE. There was no statistically significant relationship between the presence of PE and gender $(\mathrm{p}=0.064)$.

Immobility was the most frequently seen risk factor for PE in patients who were diagnosed with PE by CTA. Multiple predisposing factors for PE were noted in $32.1 \%$ of these patients. No risk factor was found to be significant correlated with the presence of PE.

Dyspnea, cough and chest pain were the most frequent symptoms among the PE patients. Fever and syncope were found to be significantly more frequent in the PE group than among patients not diagnosed with PE (Table 2).

Tachycardia, signs of DVT (including pain, swelling and tenderness, etc.) and tachypnea were the most frequent physical examination findings. There was a statistically significant relationship between tachycardia, hypotension, signs of DVT and the presence of PE ( $p=0.0001$, 0.008 and 0.0001 respectively) (Table 2 ). 
Table 2. Symptoms and physical examination findings in relation to the presence of PE

\begin{tabular}{|c|c|c|c|}
\hline $\begin{array}{l}\text { Symptoms and physical } \\
\text { examination findings }\end{array}$ & $\begin{array}{l}\text { PE present in CTA } \\
(n=39)(\%)\end{array}$ & $\begin{array}{l}\text { No PE in CTA } \\
(n=157)(\%)\end{array}$ & $p$-value \\
\hline Dyspnea & $32 / 143(22.4)$ & $111 / 143$ (77.6) & $0.108^{*}$ \\
\hline Palpitation & $5 / 29(17.2)$ & $24 / 29(82.8)$ & $0.460^{*}$ \\
\hline Hemoptysis & $2 / 12(16.7)$ & $10 / 12(83.3)$ & $0.560^{*}$ \\
\hline Cough & $14 / 39(35.8)$ & $25 / 39(64.2)$ & $0.530^{*}$ \\
\hline Syncope & $8 / 13(61.5)$ & $5 / 13(38.5)$ & $0.001^{*}$ \\
\hline Tachypnea & 19/83 (22.9) & $64 / 83(77.1)$ & $0.235^{*}$ \\
\hline
\end{tabular}

${ }^{*} x^{2}$ test.

Table 3. The presence of PE in relation to clinical scores according to the 4 systems

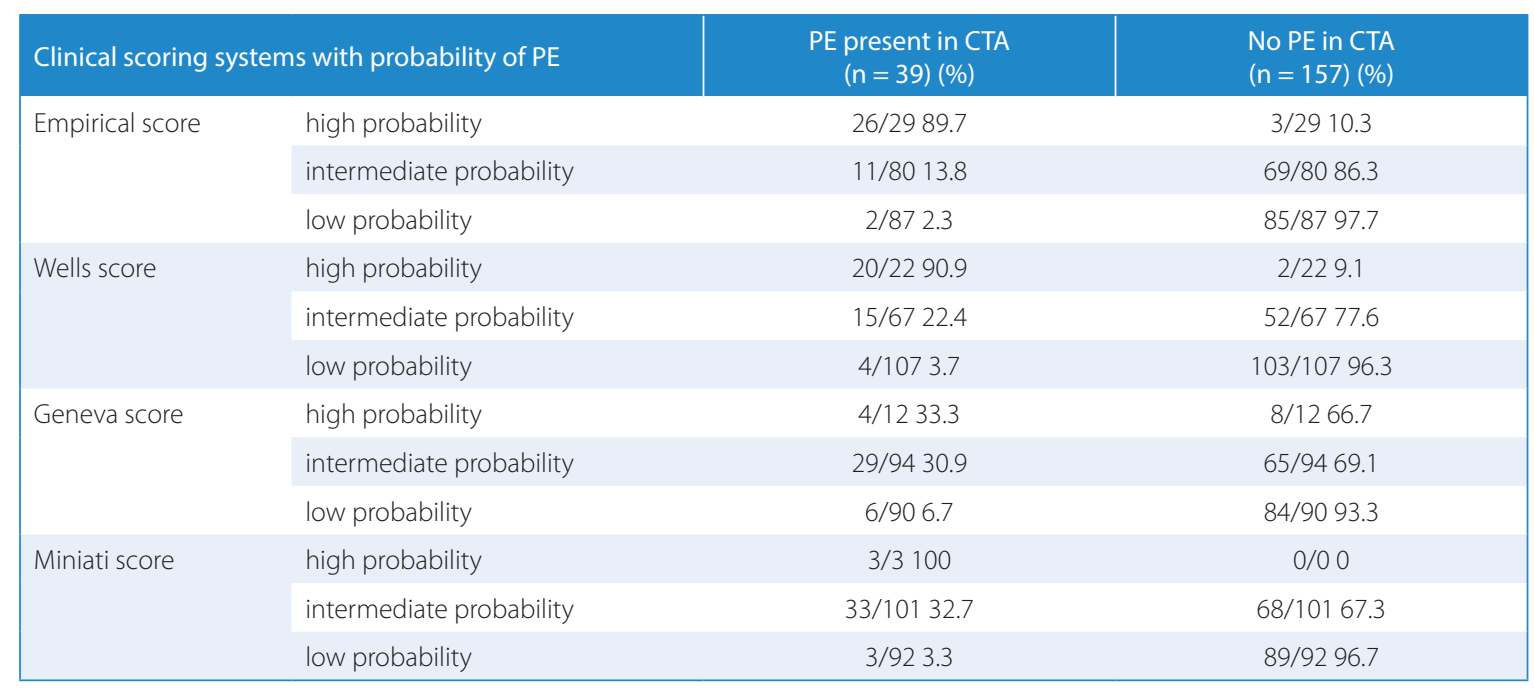

Among the patients who had PE, empirical scoring indicated that $26(89.7 \%)$ had a high clinical probability of having PE, 11 (13.8\%) had an intermediate probability and $2(2.3 \%)$ had a low clinical probability. According to the Wells scoring criteria, 20 PE patients (90.9\%) had a high probability, $15(22.4 \%)$ had an intermediate probability and 4 (3.7\%) had a low probability of having PE. According to Geneva scoring only 4 PE patients (3.7\%) had a high clinical probability. According to Miniati scoring only 3 patients had a high clinical probability of $\mathrm{PE}$, and all 3 (100\%) indeed had PE (Table 3).

The sensitivity of the various scoring systems for PE was found as $94.9 \%$ for empirical scoring, $89.7 \%$ for the Wells criteria, $84.6 \%$ for Geneva scoring, and $92.3 \%$ for the Miniati system. The specificity was $54.1,65.6,53.5$ and $56.7 \%$ respectively; PPV was $33.9,39.3,31.1$ and 34.6 respectively; while NPV was 97.7, 96.3, 93.3 and 96.7\% respectively. It was remarkable that empirical scoring had the highest sensitivity, while the Wells method was found to have the highest specificity.

D-dimer levels were analyzed in 141 patients. They were high in 117 patients (83\%), with a cut-off value of $500 \mathrm{ng} / \mathrm{mL}$ according to the ELISA test. D-dimer sensitivity was found to be $96.3 \%$; specificity was $20.2 \%$; PPV was 22.2\% and NPV was 95.3\%.

The area under the ROC curve was calculated as 0.76 , $0.69,0.75,0.74$ and 0.82 respectively for the Wells scoring system, Geneva scoring, empirical scoring, Miniati scoring and the Wells system with a cut-off score of 4 (Fig. 1).

Normal serum D-dimer levels in conjunction with low probability scores for PE were evaluated together in every scoring system (Table 4); sensitivity was found to be $100 \%$ in all methods. Wells scoring with a cut-off score of 4 had a specificity of $56.1 \%$. There was no PE in any patient with a normal D-dimer level and a low probability score in any scoring system. 


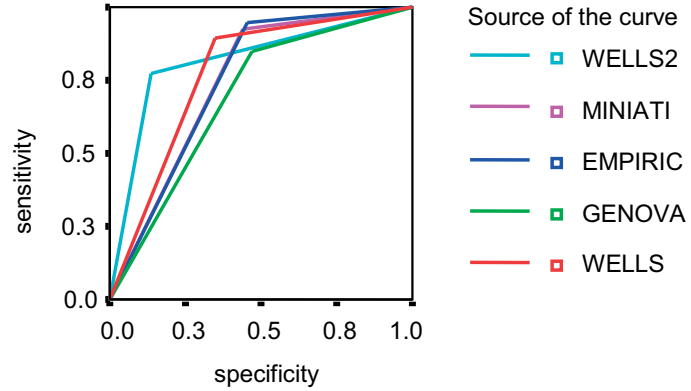

Fig. 1. Scoring systems with the area under the ROC curves. Diagonal segments are produced by ties

Wells2 - Wells system with a cut-off score of 4

Table 4. The presence of PE according to clinical scores combined with D-dimer levels

\begin{tabular}{|l|r|r|}
\hline & \multicolumn{2}{|c}{$\begin{array}{c}\text { PE in BT } \\
\text { angiography }\end{array}$} \\
\cline { 2 - 3 } and scoring systems & PE (+) & PE (-) \\
\hline D-dimer level and empiric score & 27 & 114 \\
The other combinations* & 27 & 101 \\
Normal D-dimer level and low probability & 0 & 13 \\
D-dimer level and Wells score & 27 & 114 \\
The other combinations* & 27 & 94 \\
Normal D-dimer level and low probability & 0 & 20 \\
D-dimer level and Geneva score & 27 & 114 \\
The other combinations* & 27 & 96 \\
Normal D-dimer level and low probability & 0 & 18 \\
D-dimer level and Miniati score & 27 & 114 \\
The other combinations* & 27 & 103 \\
Normal D-dimer level and low probability & 0 & 11 \\
D-dimer level and Wells score with a cut off score of 4 & 19 & 41 \\
High D-dimer level \& Wells score $>4$ & 19 & 18 \\
Normal D-dimer level \& Wells score $\leq 4$ & 0 & 23 \\
\hline
\end{tabular}

* The other combinations: Normal D-dimer level and intermediate/high probability; high D-dimer level and any probability.

\section{Discussion}

Symptoms or physical examination parameters can guide the clinician in the assessment of PE. In the present study, dyspnea and chest pain were the most common symptoms, while tachypnea and tachycardia were the most frequent physical examination findings in patients admitted to the emergency department with suspected PE. These results were similar to other studies. ${ }^{12}$ Tachycardia, hypotension and signs of DVT, symptoms like syncope and fever were found to be higher in the PE group, which was statistically significant. Although symptoms and signs are not considered specific for a PE diagnosis, they have an important role in predicting PE. ${ }^{13}$

Immobility, malignancy and recent surgery were found to be the most frequent risk factors for PE. ${ }^{14}$ In the present study, there were no statistically significant differences between patients with or without PE regarding predispos- ing factors. The presence of multiple risk factors (32.1\%) in the patients in the study group is thought to be the reason for this result.

As over-investigation of PE in emergency departments still remains an important problem and PE has a high mortality without treatment, early diagnosis is very important. There are some diagnostic procedures, such as pulmonary angiography or CTA which are invasive and expensive, and are not available in all medical centers. ${ }^{1,15,16}$ Clinical scoring systems are used to optimize the management of patients with suspected PE and to help select patients who should be examined further for PE. Clinical assessments have been highlighted by several prospective studies. The most important and prominent clinical evaluation systems for PE are the empirical, Wells, Geneva and Miniati pre-tests.

The empirical scoring system is one of the clinical assessments used for evaluating the presence of PE. In one study PE diagnostic rates were found to be 89.9, 11.7 and $2.8 \%$ of the cases ranked by empirical scoring as high, intermediate and low probability respectively. ${ }^{17}$ In the present study, the PE diagnostic rates were $89.7 \%$ of the high probability cases, $13.8 \%$ of the intermediate probability cases and $2.3 \%$ of the low probability cases. Gülcü et al. found empirical assessment more sensitive and useful than other pre-tests in the diagnostic management of PE. ${ }^{18}$ The results of the present study show that that the empirical scoring system had the highest sensitivity and NPV, which makes it an effective method for patient selection for further evaluation with CTA.

The Prospective Investigation of Pulmonary Embolism Diagnosis (PIOPED) study was an important prospective study which found that $68 \%$ of the high probability cases, $30 \%$ of the intermediate probability cases and $9 \%$ of the low probability cases had PE. ${ }^{12}$ Wells et al. developed a prediction system by scoring clinical data from the PIOPED study. PE rates were reported to be $66.7 \%$, $20.5 \%$ and $3.6 \%$ in high, intermediate and low probability groups, respectively, according to the Wells criteria. ${ }^{2}$ In the present study, $90.9 \%$ of the cases rated high probability according to the Wells system had PE, as did $22.4 \%$ of those rated intermediate and $3.7 \%$ of those rated low probability. Although some studies have suggested that the Wells score does not guarantee exclusion of PE, especially in older patients, this pre-test has been reported to be more useful than other tests in the diagnosis of PE in respiratory emergencies in most studies. ${ }^{6,19}$ In the present study it was remarkable that the Wells method was found to have the highest specificity and PPV of all 4 pre-tests.

The Geneva scoring system had been developed by Wicki et al; $81 \%$ of the high probability cases according to Geneva scoring had PE, as did 38\% of the intermediate probability cases and $10 \%$ of the low probability cases. ${ }^{9}$ In one study the Geneva score was reported to be one of the most useful diagnostic tools for PE. ${ }^{20}$ The present study showed 33.3, 30.9 and $6.7 \%$ rates of PE in the Geneva high, intermediate 
and low probability groups respectively, which meant that this scoring system had lower specificity, sensitivity, PPV and NPV than the other 3 scoring systems.

The Prospective Investigative Study of Acute Pulmonary Embolism Diagnosis (PISAPED) study, which used a scoring system called the Miniati method, included acute symptoms of PE, electrocardiographic signs and radiological findings. ${ }^{10} \mathrm{PE}$ was diagnosed in $91 \%$ of the cases classed as high probability according to the Miniati method, $47 \%$ of the intermediate probability cases and $9 \%$ of the low probability cases. ${ }^{10}$ In the present study PE was found in $100 \%$ of those the Miniati method rated as high probability, $32.7 \%$ of those rated intermediate and $3.3 \%$ of those rated low probability. As the $100 \%$ high probability comprised only 3 patients, the Miniati scoring system does not seem to be a strong assessment tool.

D-dimer levels are an important test for excluding PE, especially with the help of clinical scoring systems. ${ }^{12}$ On their own, D-dimer levels have high sensitivity and NPV, but low specificity and PPV; the low specificity of $35-40 \%$ limits the clinical usefulness of D-dimer levels. ${ }^{20,21}$ Low D-dimer levels have been used as a guide for excluding patients with low or intermediate probability of suspected PE. ${ }^{2,12,22}$ Some studies have shown that the combination of normal D-dimer levels and low clinical probability was safe for the exclusion of PE; van Belle et al. documented a 3-month incidence of venous thromboembolism (VTE) of only $0.5 \%$ of after excluding PE with an unlikely clinical score and normal D-dimer levels. ${ }^{23-26}$ In the present study the combination of normal serum Ddimer levels and low probability in all the scoring methods had a result of $100 \%$ sensitivity, which means no PE was detected in these patients. In the subgroup of patients with normal D-dimer levels and low/intermediate probability, sensitivity was $100 \%$ only in the Wells and empirical scoring systems. These results show that normal D-dimer levels and low clinical probability in all systems, and low/ intermediate probability in the Wells or empirical scoring methods, are useful combinations for ruling out PE.

Wells et al. updated their scoring system by creating 2 categories (PE likely or unlikely) with a cut-off score of 4 points to improve the simplicity of the method, which was named as modified Wells score. ${ }^{23}$ In a previous study, modified Wells scores and D-dimer levels were evaluated together, which was found to be more successful in excluding PE. ${ }^{23,26,27}$ In the present study the specificity of the dichotomized Wells system combined with D-dimer levels was $56.1 \%$, which was the highest of all the clinical scoring systems combined with D-dimer levels. This result shows that the dichotomized version of the Wells system combined with D-dimer levels may be an important method for the diagnosis of PE.

The Wells assessment system, which was found to be useful for diagnosis of PE in the present study, is one of the most popular methods currently used in emergency departments. Data showing that the combination of nor- mal serum D-dimer levels and low probability of PE in scoring systems mostly exclude the diagnosis of PE have also been reported in several studies. ${ }^{23-25}$ Empirical scoring, which is not as popular as Wells scoring nowadays, was also found to be a useful method for patients with suspected PE. The present study indicates that the empirical method can be used frequently for patient selection in the emergency department.

The present study has some potential limitations. First of all, it is retrospective. The authors believe that it is objective, as patients with the suspicion of PE were included in the study, not only patients with PE. A prospective study in which the patients are followed up for a few months and evaluated with a CTA for the presence of PE may be more objective in comparing the clinical scoring systems. Another limitation was the small number of patients in some of the subgroups.

\section{Conclusions}

Clinical scoring systems may be a guide for the diagnostic management of patients in whom PE is suspected. The empirical scoring system and Wells criteria have been found to be the most useful methods for patient selection. Further evaluation of serum D-dimer levels in combination with clinical scores could determine the group of patients in whom CTA should be performed. The combination of normal serum D-dimer levels and low probability scores in any clinical scoring system is a reliable method for the exclusion of PE, which can avoid subjected these patients to CTA. The combination of the dichotomized Wells score and D-dimer level seems to be important in diagnosing PE. Evaluation using clinical scoring systems and D-dimer levels is practical for use in the emergency department to rule out a diagnosis of PE and to guide the diagnostic algorithm for patient selection for further evaluation.

\section{References}

1. Dalen JE. Pulmonary embolism: What have we learned since Virchow? Chest. 2002;122:1440-1446.

2. Wells PS, Ginsberg JS, Anderson DR, et al. Use of a clinical model for safe management of patients with suspected pulmonary embolism. Ann Intern Med. 1998;129:997-1005.

3. Schoepf UJ, Goldhaber SZ, Costello P. Spiral computed tomography for acute pulmonary embolism. Circulation. 2004;109:2160-2167.

4. Johnson MS. Current strategies for the diagnosis of pulmonary embolus. J Vas Interv Radiol. 2002;13:13-23.

5. Moores LK, Collen JF, Woods KM, Shorr AF. Practical utility of clinical prediction rules for suspected acute pulmonary embolism in a large academic institution. Thromb Res. 2004;113:1-6.

6. Tsimogianni AM, Rovina N, Porfyridis I, et al. Clinical prediction of pulmonary embolism in respiratory emergencies. Thromb Res. 2011;127(5):411-417.

7. Penaloza A, Mélot C, Dochy E. Comparison of the Wells score with the simplified revised Geneva score for assessing pretest probability of pulmonary embolism. Thromb Res. 2007;120(2):173-179. Epub 2006 Oct 20.

8. Hyers TM. Venous thromboembolism. Am J Respir Crit Care Med. 1999;159:1-14. 
9. Wicki J, Perneger TH, Jumnod A, Bounameaux H, Perrier A. Assessing clinical probability of pulmonary embolism in the emergency ward. Arch Intern Med. 2001;161:92-97.

10. Miniati M, Prediletto R, Formichi B, et al. Accuracy of clinical assessment in the diagnosis of pulmonary embolism. Am J Respir Crit Care Med. 1999;159:864-871.

11. Perrier A, Desmarais S, Goehring C, et al. D-dimer testing for suspected pulmonary embolism in outpatients. Am J Respir Crit Care Med. 1997;156:492-496.

12. The PIOPED Investigators. Value of ventilation/perfusion scan in acute pulmonary embolism: Results of the prospective investigators of pulmonary embolism diagnosis (PIOPED). JAMA. 1990;263: 2753-2759.

13. Stein PD, Terrin ML, Hales CA, Palevsky HI, Saltzman HA, Thompson BT. Clinical, laboratory, roentgenographic, and electrocardiographic findings in patients with acute pulmonary embolism and no pre-existing cardiac or pulmonary disease. Chest. 1991;100(3):598-603.

14. Anderson FA Jr., Spencer FA. Risk factors for venous thromboembolism. Circulation. 2003;107:19-16.

15. Aydoğdu M, Topbaşi Sinanoğlu N, Doğan NO, et al. Wells score and pulmonary embolism rule out criteria in preventing over investigation of pulmonary embolism in emergency departments. Tuberk Toraks. 2014;62(1):12-21.

16. Fedullo PF, Tapson VF. Clinical practice. The evaluation of suspected pulmonary embolism. N Engl J Med. 2003;349(13):1247-1256.

17. Ergün $\mathrm{P}$, Oran D, Erdoğan $\mathrm{Y}$, Biber C, Caglar A. Clinical probability and noninvasive methods in the diagnosis of pulmonary thromboembolism. Solunum Hastalıkları. 2004;15:8-14.

18. Gülcü A, Akkoçlu A, Yilmaz E, Oztürk B, Osma E, Sengün B. Comparison of clinical assessments with computerized tomography pulmonary angiography results in the diagnosis of pulmonary embolism. Tuberk Toraks. 2007;55(2):174-181.
19. Schouten HJ, Geersing GJ, Oudega R, van Delden JJ, Moons KG, Koek HL. Accuracy of the wells clinical prediction rule for pulmonary embolism in older ambulatory adults. J Am Geriatr Soc. 2014; 62(11):2136-2141.

20. Blondon M, Le Gal G, Righini M. Diagnostic strategy and comparison of clinical scores for pulmonary embolism. Rev Med Interne. 2010;31(11):742-749. Epub 2010 Aug 25.

21. Kelly J, Rudd A, Lewis RR, Hunt BJ. Plasma D-dimers in the diagnosis of venous thromboembolism. Arch Intern Med. 2002;162:747-756.

22. Righini M, Aujesky D, Roy PM, et al. Clinical usefulness of D-dimer depending on clinical probability and cutoff value in outpatients with suspected pulmonary embolism. Arch Intern Med. 2004; 164(22):2483-2487.

23. Wells PS, Anderson DR, Rodger M, et al. Derivation of a simple clinical model to categorize patients' probability of pulmonary embolism: Increasing the model's utility with the SimpliRED D-dimer. Thromb Haemost. 2000;83:416-420.

24. Kruip MJ, Slob MJ, Schijen JH, van der Heul C, Büller HR. Use of a clinical decision rule in combination with $\mathrm{D}$-dimer concentration in diagnostic workup of patients with suspected pulmonary embolism: a prospective management study. Arch Intern Med. 2002;162: 1631-1635.

25. Ten Wolde M, Hagen PJ, MacGillavry MR, et al. Non-invasive diagnostic work-up of patients with clinically suspected pulmonary embolism: Results of a management study. J Thromb Haemost. 2004;2:1110-1117.

26. Van Belle A, Büller HR, Huisman MV, et al. Effectiveness of managing suspected pulmonary embolism using an algorithm combining clinical probability, D-dimer testing, and computed tomography. JAMA.2006;295:172-179.

27. Wolf SJ, McCubbin TR, Feldhaus KM, Faragher JP, Adcock DM. Prospective validation of Wells criteria in the evaluation of patients with suspected pulmonary embolism. Ann Emerg Med. 2004;44: 503-510. 